\title{
Phantom evolving wormholes with big rip singularities
}

\author{
Mauricio Cataldd* \\ Departamento de Física, Facultad de Ciencias, Universidad del Bío-Bío, \\ Avenida Collao 1202, Casilla 5-C, Concepción, Chile. \\ Paola Mezat \\ Departamento de Física, Universidad de Concepción, \\ Casilla 160-C, Concepción, Chile.
}

(Dated: September 27, 2018)

\begin{abstract}
We investigate a family of inhomogeneous and anisotropic gravitational fields exhibiting a future singularity at a finite value of the proper time. The studied spherically symmetric spacetimes are asymptotically Friedmann-Robertson-Walker at spatial infinity and describe wormhole configurations filled with two matter components: one inhomogeneous and anisotropic fluid and another isotropic and homogeneously distributed fluid, characterized by the supernegative equation of state $\omega=p / \rho<-1$. In previously constructed wormholes, the notion of the phantom energy was used in a more extended sense than in cosmology, where the phantom energy is considered a homogeneously distributed fluid. Specifically, for some static wormhole geometries the phantom matter was considered as an inhomogeneous and anisotropic fluid, with radial and lateral pressures satisfying the relations $p_{r} / \rho<-1$ and $p_{l} \neq p_{r}$, respectively. In this paper we construct phantom evolving wormhole models filled with an isotropic and homogeneous component, described by a barotropic or viscous phantom energy, and ending in a big rip singularity. In two of considered cases the equation of state parameter is constrained to be less than -1 , while in the third model the finite-time future singularity may occur for $\omega<-1$, as well as for $-1<\omega \leq 1$.
\end{abstract}

PACS numbers: 04.20.Jb, 04.70.Dy,11.10.Kk

\section{INTRODUCTION}

Recent astrophysical observations indicate that our Universe is currently in accelerating expansion [1, 2]. This discovery has stimulated an intensive study of models where a large number of possible cosmological mechanisms have been proposed to explain the origin of the current acceleration. In the framework of general relativity, a quantitative analysis shows that a mysterious component of energy, dubbed dark energy, is responsible for the origin of this cosmic phenomenon and it starts dominating the matter content dynamics only at recent times, being irrelevant at earlier stages of the evolution. Many dark energy models have been proposed to solve this fundamental problem of cosmological physics, such as $\Lambda$-CDM model [3], quintessence models [2, 4], k-essence models [5], phantom models [6], etc.

These new advances in cosmology allow us to consider new type of singularities, besides already considered standard singularities such as Big-Bang and Big-Crunch [7]. This non-standard type of singularities occurs at a finite value of cosmological time and are included in the following descriptive classification: the singularity is a Big Rip when the scale factor, energy density and pressure go to infinity in a finite proper time, and is a sudden singularity when at a finite value of time and scale factor,

\footnotetext{
*mcataldo@ubiobio.cl

†paolameza@udec.cl
}

curvature or one of its higher derivatives blow up [8].

The main motivation of this type of singularities comes from phantom cosmological models. In the framework of Friedmann-RobertsonWalker (FRW) cosmologies filled with two matter contents and dominated by a phantom type fluid, satisfying a non-dissipative barotropic equation of state, this new type of singularities can be completely classified in the following four types: type I for a Big Rip singularity; type II for a sudden singularity defined by a finite energy density and diverging pressure; type III for diverging energy density and pressure at a finite value of the scale factor, and the type IV for finite curvature components and diverging higher derivatives of $H$ [9].

On the other hand, since the pioneering works by Morris and Thorne [10], the study of wormholes has become one of the most popular and intensively studied topics in relativistic physics, where most of the efforts are directed to study Lorentzian wormholes, in the framework of classical general relativity, sustained by an exotic matter with negative energy density. These models include both static [11] and evolving relativistic versions [12], sustained by a single fluid component. The interest has been mainly devoted to traversable wormholes, which have no horizons, allowing two-way passage through them [13].

For static wormholes the fluid requires the violation of the null energy condition (NEC), while in Einstein gravity there are nonstatic Lorentzian wormholes which do not require WEC violating matter to sustain them. Such wormholes may exist for arbitrarily small or large intervals of time [14] or even satisfy the dominant energy con- 
dition (DEC) in the whole spacetime [15, 16]. One can consider also dynamic wormhole spacetimes filled with two fluids, just like it is required in cosmology where such two-fluid models are widely considered today in order to explain the observed accelerated expansion of the Universe [17].

Wormhole spacetimes filled with phantom type matter were considered before [18]. Specifically, spherically symmetric static wormholes were studied, sustained by a phantom type matter (or super-quintessence) with anisotropic pressure. In these static models the notion of the phantom energy is used in a more extended sense than in cosmology since, strictly speaking, the phantom matter is a homogeneously distributed fluid, and for these non-dynamic wormhole models an inhomogeneous and anisotropic matter component is used, having for the radial and lateral pressures $p_{r}<-1$ and $p_{l} \neq p_{r}$, respectively [19]. This type of extended phantom-like matter was also used in dynamical wormhole models [20].

The time evolution of wormhole geometries in a Friedmann universe exhibiting a Big Rip singularity were previously studied. However the literature on this topic is not extensive. For example, in Ref. 21] the author considers some accelerated higher-dimensional cosmologies with a traversable static wormhole, dominated by a timedependent cosmological constant, and ending at a Big Rip. The studied Big Rip solutions have an exponential scale factor. In addition, the authors of Ref. [22] consider two different wormhole models, modeled by a thin spherical shell accreting the phantom fluid.

It is interesting to note that in this context, it has recently proposed that it is possible that the universe could avoid the big rip singularity with the occurrence of a big trip [23, 24], which is a cosmological event that may appear during the evolution of a wormhole embedded in a FRW universe approaching the Big Rip singularity. In this case, the wormhole accreting phantom matter expands faster than the background FRW universe, and the radius of the wormhole throat diverges before the Big Rip is reached. In this scenario, the wormhole engulfs the entire universe, which will reappear from the other wormhole throat [22].

In the present paper we intend to study evolving wormholes filled with two matter components, where one of them is an isotropic homogeneously distributed phantom fluid characterized the supernegative equation of state $\omega=p / \rho<-1$, and presenting a future singularity at a finite value of the proper time.

The organization of the paper is as follows: In Sec. II we present the dynamical field equations for wormhole models with a matter source composed of an ideal isotropic cosmic fluid and an anisotropic and inhomogeneous one. In Sec. III expanding wormholes filled with a barotropic dark energy and phantom fluids are studied. We discuss explicit models ending at a finite-time future singularity. In Sec. IV viscous expanding wormholes are discussed. Models that may evolve to a finite-time future singularity are considered, and in Sec. V we conclude with some remarks.

\section{FIELD EQUATIONS}

In this paper we shall make use of some previously obtained results by one of the authors in Ref. [16]. Let us state the main result obtained in that paper, concerning with solutions containing two fluids and admitting spherical symmetry in the framework of the Einstein gravity theory.

Taking the metric

$$
d s^{2}=-d t^{2}+a(t)^{2}\left(\frac{d r^{2}}{1-k r^{2}-\frac{b(r)}{r}}+r^{2} d \Omega^{2}\right),
$$

in comoving coordinates, filled with the anisotropic and inhomogeneous fluid $\rho_{i n}(t, r)$, and the isotropic and homogeneous fluid $\rho(t)$, the Einstein equations are given by

$$
\begin{array}{r}
3 H^{2}+\frac{3 k}{a^{2}}+\frac{b^{\prime}}{a^{2} r^{2}}=\kappa \rho_{i n}(t, r)+\kappa \rho(t)+\Lambda,(2) \\
-\left(2 \frac{\ddot{a}}{a}+H^{2}+\frac{k}{a^{2}}\right)-\frac{b}{a^{2} r^{3}}=\kappa p_{r}(t, r)+\kappa p(t)-\Lambda,(3) \\
-\left(2 \frac{\ddot{a}}{a}+H^{2}+\frac{k}{a^{2}}\right)+\frac{b-r b^{\prime}}{2 a^{2} r^{3}}=\kappa p_{l}(t, r)+\kappa p(t)-\Lambda,(4)
\end{array}
$$

where $d \Omega^{2}=d \theta^{2}+\sin ^{2} \theta d \varphi^{2}, \kappa=8 \pi G ; \Lambda$ is the cosmological constant, $a(t)$ is the scale factor, $k=-1,0,1$; $H=\dot{a} / a$; and an overdot and a prime denote differentiation $d / d t$ and $d / d r$ respectively.

In this case the 4-velocity of the fluids is given by the timelike vector $u^{\alpha}=(1,0,0,0)$, and the radial and tangential pressures obey the barotropic state equations

$$
\begin{array}{r}
p_{r}(t, r)=\omega_{r} \rho_{i n}(t, r), \\
p_{l}(t, r)=\omega_{l} \rho_{i n}(t, r),
\end{array}
$$

with constant state parameters $\omega_{r}$ and $\omega_{l}$.

Note that the essential characteristics of a wormhole geometry are encoded in the spacelike section of the metric (1). It is clear that this metric becomes a zero-tidal force static wormhole if $a(t) \rightarrow$ const, and as $b(r) \rightarrow k r^{3}$ it becomes a flat FRW metric for $k=0$, a closed FRW metric for $k=1$, and an open FRW metric for $k=-1$.

It can be shown that Eqs. (10)-(4) may be rewritten in the form [16]

$$
\begin{gathered}
d s^{2}=d t^{2} \\
-a(t)^{2}\left(\frac{d r^{2}}{1-k r^{2}+\kappa C \omega_{r} r^{-1-1 / \omega_{r}}}+r^{2} d \Omega^{2}\right), \\
3 H^{2}+\frac{3 k}{a^{2}}=\kappa \rho(t)+\Lambda, \\
\dot{\rho}+3 H(\rho+p)=0,
\end{gathered}
$$


where $C$ is an integration constant, and the inhomogeneous and anisotropic cosmic fluid is given by

$$
\begin{aligned}
p_{r} & =\omega_{r} \rho_{i n}, \\
p_{l} & =-\frac{1}{2}\left(1+\omega_{r}\right) \rho_{i n}, \\
\rho_{i n}(t, r) & =\frac{C r^{-3-1 / \omega_{r}}}{a^{2}(t)} .
\end{aligned}
$$

Here, the constraint $\omega_{r}+2 \omega_{l}+1=0$ for Eqs. (5) was used. From these expressions we conclude that if $\omega_{r}>0$ or $\omega_{r}<-1$ the obtained gravitational configurations are asymptotically FRW solutions at spatial infinity.

In conclusion, the main result of the Ref. [16] is that the evolution of the scale factor $a(t)$ in the metric (6) is governed by the standard Friedmann equations (7) and (8), and it is determined by the fluid $\rho(t)$. This matter component may be in principle an ideal barotropic fluid or any other cosmic fluid satisfying the requirements of isotropy and homogeneity.

\section{EXPANDING WORMHOLE UNIVERSES FILLED WITH A BAROTROPIC DARK ENERGY AND PHANTOM FLUIDS}

In this section we shall consider that the isotropic and homogeneous matter component is described by a barotropic phantom energy $\rho(t)$ with an equation of state of the form

$$
p(t)=\omega \rho(t)
$$

where the constant state parameter $\omega$ satisfies the constraint $\omega<-1$. In general Eq. (12) allows us to consider a barotropic matter component describing standard matter for $\omega \geq 0$, a dark energy fluid for $-1<\omega<-1 / 3$, and super-quintessence for $\omega<-1$, just like it is defined in cosmology.

From now on in this section, we shall consider solutions with $k=\Lambda=0$ and $\kappa=8 \pi G=1$. Thus in this case, from Eqs. (7) and (8), we obtain that the scale factor is given by $a(t)=D(F+(3 / 2)(\omega+1) t)^{2 /(3(\omega+1))}$, where $D$ and $F$ are constants of integration. This scale factor we shall rewrite as

$$
a(t)=a_{0}\left(1+\frac{3}{2} H_{0}(\omega+1) t\right)^{2 /(3(\omega+1))},
$$

and the energy density in the form

$$
\rho(t)=\frac{\rho_{0}}{\left(1+\frac{3}{2} H_{0}(\omega+1) t\right)^{2}},
$$

where $\rho_{0}=3 H_{0}^{2}$, in order to have $a\left(t_{0}=0\right)=a_{0}>0$ and $H\left(t_{0}=0\right)=H_{0}>0$.

It is easy to verify that in this case the metric (6) becomes

$$
d s^{2}=d t^{2}-a_{0}^{2}\left(1+\frac{3}{2} H_{0}(\omega+1) t\right)^{4 /(3(\omega+1))} \times
$$

$$
\left(\frac{d r^{2}}{1-\left(\frac{r}{r_{0}}\right)^{-\left(1+\omega_{r}\right) / \omega_{r}}}+r^{2}\left(d \theta^{2}+\sin ^{2} \theta d \varphi^{2}\right)\right),
$$

and the anisotropic and inhomogeneous energy density is given by

$$
\rho_{i n}(t, r)=-\frac{\left(\frac{r}{r_{0}}\right)^{-\left(1+3 \omega_{r}\right) / \omega_{r}}}{r_{0}^{2} \omega_{r} a_{0}^{2}\left(1+\frac{3}{2} H_{0}(\omega+1) t\right)^{4 /(3(\omega+1))}} .
$$

The metric (15) represents an evolving wormhole with a throat located at $r_{0}$ for $\omega_{r}<-1$ and $\omega_{r}>0$ [16] and is asymptotically a flat FRW universe.

It is well known that in general to keep a wormhole open exotic matter with a negative energy density at the throat is needed [10]. However, there are examples of evolving wormholes satisfying the DEC in the whole spacetime [15, 16], which implies that the energy density is positive everywhere. In the studied here solutions the branch with $\omega_{r}>0$ is characterized by a positive radial pressure $p_{r}$, and negative $\rho_{i n}$ and $p_{l}$. But, there are also wormholes which avoid the usual exotic matter requirements for wormholes. The branch with $\omega_{r}<-1$ has a positive energy density and lateral pressure, while the radial pressure is negative and larger in magnitude than the energy density. Specifically, the total matter content

$$
\rho_{T}(t, r)=\rho(t)+\rho_{i n}(t, r)
$$

for these models is determined by Eqs. (14) and (16). Thus, for any value of the state parameters $\omega$ and $\omega_{r}<0$ we have that always $\rho_{T} \geq 0$. For $\omega_{r}>0$ we can have in general time intervals where the total energy is positive or negative. In effect, for $\omega>-1 / 3$ the wormhole model starts with a positive total energy density (since for a fixed value $r=$ const the isotropic component dominates over the another one), then decreases till zero at certain $t_{0}$, and becomes negative for $t>t_{0}$. For $\omega<-1 / 3$ the total energy density starts negative, then increases till zero at certain $t_{0}$, and becomes positive for $t>t_{0}$. If $\omega=-1 / 3$ both components of total energy density (17) behave as $\sim 1 / a^{2}$, and the expansion occurs at a constant velocity.

In order to have accelerated (decelerated) expansion we must consider $\omega<-1 / 3(\omega>-1 / 3)$. Notice that at $t=0$ we have that the total energy density is given by

$$
\rho_{T}(0, r)=\rho_{0}-\frac{\left(\frac{r}{r_{0}}\right)^{-\left(1+3 \omega_{r}\right) / \omega_{r}}}{r_{0}^{2} \omega_{r} a_{0}^{2}} .
$$

We can see from Eq. (18) that if the values of $\omega_{r}$ are constrained to be in the ranges $\omega_{r}<-1 / 3$ and $\omega_{r}>$ 0 , then the relation $\rho(0)>\rho_{\text {in }}(0, r)$ is fulfilled for $r>$ $\left(-r_{0}^{1+1 / \omega_{r}} \omega_{r}^{-1} a_{0}^{-2} \rho_{0}^{-1}\right)^{\omega_{r} /\left(1+3 \omega_{r}\right)}$. If the last inequality is not fulfilled, then at $t=0$ the inhomogeneous component 


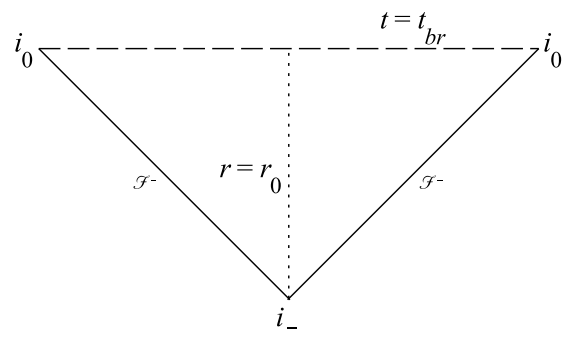

FIG. 1: Penrose diagram for an evolving wormhole with $\omega_{r}<$ -1 and $\omega<-1$. The dotted line is the wormhole throat and the dashed line represents the future Big Rip singularity.

begins dominating. If $-1 / 3<\omega_{r}<0$ we have that $\rho_{i n}(0, r)>\rho(0)$ for $r>\left(-r_{0}^{1+1 / \omega_{r}} \omega_{r}^{-1} a_{0}^{-2} \rho_{0}^{-1}\right)^{\omega_{r} /\left(1+3 \omega_{r}\right)}$.

For these models it is useful to state that if the isotropic perfect fluid satisfies the DEC, i.e. $-1<\omega<1$, we can rescale the cosmological time so that $1+\frac{3}{2} H_{0}(\omega+$ $1) t \longrightarrow t$ and the solution for the scale factor takes the standard form $a(t)=a_{0} t^{2 /(3(\omega+1))}$, with the isotropic energy density given by $\rho(t)=\rho_{0} / t^{2}$.

In order to study these models during a phantom evolution we must consider the values $\omega<-1$. In this case we have that $1+\frac{3}{2} H_{0}(\omega+1) t \equiv 1-\frac{3}{2} H_{0}|\omega+1| t$, so during the cosmic evolution this expression may vanish at some value of time $t>0$. Thus we conclude that if $\omega<-1$ we have a future singularity at a finite value of the proper time

$$
t_{b r}=-\frac{2}{3 H_{0}(\omega+1)}>t_{0}=0
$$

since $a(t) \longrightarrow \infty, \rho(t) \longrightarrow \infty$ and $p \longrightarrow-\infty$ at $t=t_{b r}$. By considering only the behavior of scale factor and the isotropic fluid we may conclude that this future singularity is of a Big Rip type.

However we must also consider the presence of the inhomogeneous matter component (16). As we have stated above in this case we have that $\rho_{i n}(t, r)>0$ for $\omega_{r}<0$, and $\rho_{i n}(t, r)<0$ for $\omega_{r}>0$. For $\omega_{r}=-1 / 3$ this matter component becomes homogeneous and isotropic one with $p_{r}=p_{l}=-\rho_{\text {in }} / 3$. Note that for $\omega>-1, \omega_{r}<-1 / 3$ and $\omega_{r}>0$ we have that $\rho_{i n}\left(t_{c}, r \longrightarrow \infty\right) \longrightarrow 0$ and $\rho_{\text {in }}\left(t \longrightarrow \infty, r_{c}\right) \longrightarrow 0$ for constant values $t_{c}$ and $r_{c}$.

For $\omega<-1, \omega_{r}<-1 / 3$ and $\omega_{r}>0$ we have that at $t=0$ the anisotropic and inhomogeneous energy density starts to evolve from the constant value $\rho_{i n}\left(0, r_{c}\right)$, where the constant $r_{c} \geq r_{0}$, and then decreases, becoming zero at $t=t_{b r}$.

As a consequence, for $t=t_{b r}$ and $\omega<-1$, we have that $a(t) \longrightarrow \infty, \rho(t) \longrightarrow \infty, p \longrightarrow-\infty, \rho_{i n}(t, r)=0$, $p_{r}(t, r)=p_{l}(t, r)=0$. This allows us to conclude that if we consider the total matter content given by Eq. (17), for $t=t_{b r}$ and $\omega<-1$, we have that $a(t) \longrightarrow \infty$, $\rho_{T}(t, r) \stackrel{\longrightarrow}{\longrightarrow}, p_{T r}(t, r) \longrightarrow-\infty, p_{T l}(t, r) \longrightarrow-\infty$, where $p_{T r}(t, r)=p(t)+p_{r}(t, r)$ and $p_{T l}(t, r)=p(t)+$ $p_{l}(t, r)$. In conclusion, the scale factor, the total energy density and the total pressures blow up at the finite time $t_{b r}$, so this finite-time future singularity is of a Big Rip type. In Fig. 1 is shown a conformal diagram of an evolving wormhole with $\omega_{r}<-1$ and a phantom energy with $w<-1$.

Lastly, let us introduce the equilibrium time defined by the condition $\rho\left(t_{e q}\right)=\rho_{i n}\left(t_{e q}, r\right)$, for wormholes models with a positive total energy density and big rip. This allows us to find $t_{e q}$ as a function of the radial coordinate $r$ :

$$
t_{e q}=\frac{2}{3 H_{0}(1+\omega)}\left[\left(-\frac{\left(\frac{r}{r_{0}}\right)^{-\frac{1+3 \omega_{r}}{\omega_{r}}}}{r_{0}^{2} \omega_{r} a_{0}^{2} \rho_{0}}\right)^{\frac{-3(1+\omega)}{2(1+3 \omega)}}-1\right]
$$

It becomes clear that in general $t_{e q}$ can take complex as well as real values. In order to have always real values of the equilibrium time the condition $\omega_{r}<0$ must be required. For wormhole models with a positive total energy density and big rip the equilibrium time is positive if $\omega_{r}<-1, \omega<-1$ and

$$
-\frac{\left(\frac{r}{r_{0}}\right)^{-\frac{1+3 \omega_{r}}{\omega_{r}}}}{r_{0}^{2} \omega_{r} a_{0}^{2} \rho_{0}}>1
$$

Thus, for $r_{0}<r<r_{*}$, where

$$
r_{*}=r_{0}\left(-r_{0}^{2} \omega_{r} a_{0}^{2} \rho_{0}\right)^{-\omega_{r} /\left(1+3 \omega_{r}\right)}
$$

we have that $t_{e q}>0$, and $t_{e q}<0$ for $r>r_{*}$. Notice that from Eqs. (18) and (21) we obtain for the total energy density the equivalent constraint $\rho_{T}(0, r)>2 \rho_{0}$, and from Eqs. (19) and (20) the condition $t_{e q}<t_{b r}$ is automatically fulfilled for $\omega_{r}<0$. By using Eqs. (20)(22) qualitative plots for wormholes defined by conditions $\omega_{r}<-1$ and $\omega_{r}>0$ are shown in Figs. 2(a) $2(\mathrm{c})$ and in Figs. 3(a) 3(c) respectively.

Now we want to study evolving wormhole models with future singularities and filled with a viscous phantom matter. This issue will be addressed in the next section.

\section{VISCOUS EVOLVING WORMHOLES}

Let us now consider wormhole models with the isotropic and homogeneous matter component described by a viscous phantom fluid. The role of the dissipative processes has been extensively considered in cosmology [25, 26], where the study is done within the framework of the standard Eckart theory of relativistic irreversible thermodynamics. Any dissipation process in a FRW cosmology is scalar, and therefore may be modeled 


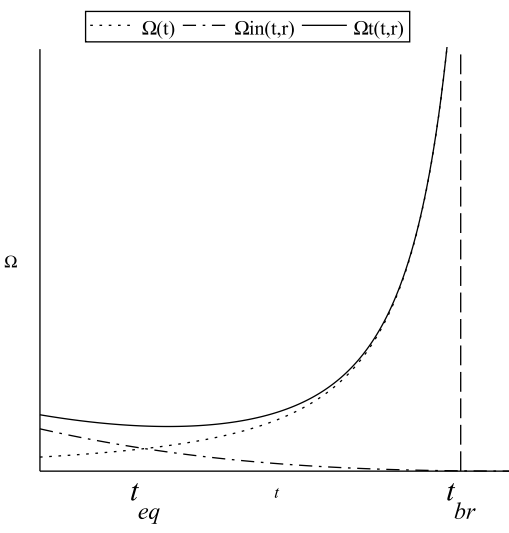

(a) $r_{0}<r_{c}<r_{*}$

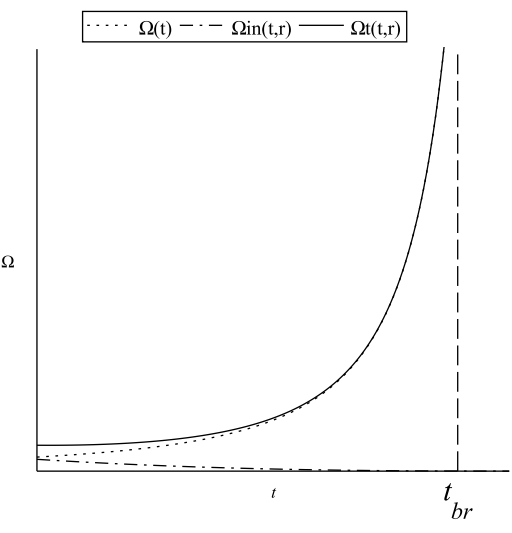

(b) $r_{c}>r_{*}$

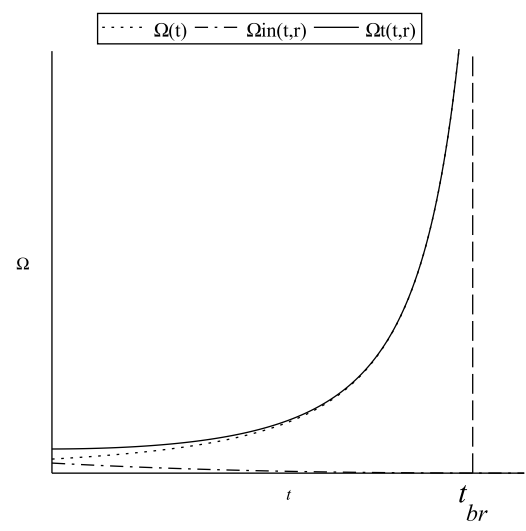

(c) $r_{c} \geq r_{0}\left(r_{*}<r_{0}\right)$

FIG. 2: These figures show, for wormhole models with $\omega_{r}<-1$ and $\omega<-1$, the qualitative behavior of the dimensionless energy density for the isotropic component $\Omega(t)$ (dotted line), anisotropic matter $\Omega_{i n}\left(t, r_{c}\right)$ (dash-dotted line) and the total matter content $\Omega_{T}\left(t, r_{c}\right)$ (solid line) as a function of the time $t$, at a constant value of the radial coordinate $r_{c}$. The comoving time varies from 0 to the big rip time $t_{b r}$. In Figs. 2(a) and 2(b) are plotted curves fulfilling the constraint (21): if $r_{0}<r_{c}<r_{*}$ the anisotropic component dominates for $0 \leq t<t_{e q}$, while the isotropic component dominates over the anisotropic one at the time interval $t_{e q}<t<t_{b r}$ (Fig. 2(a)); on the other hand if $r_{c}>r_{*}$ the isotropic component always dominates over the anisotropic for $0 \leq t<t_{b r}$ (Fig. 2(b)). In Fig. 2(c) the constraint (21) is not fulfilled, thus always the isotropic component dominates over the anisotropic one for $0 \leq t<t_{b r}$ and any constant value $r_{c} \geq r_{0}$.

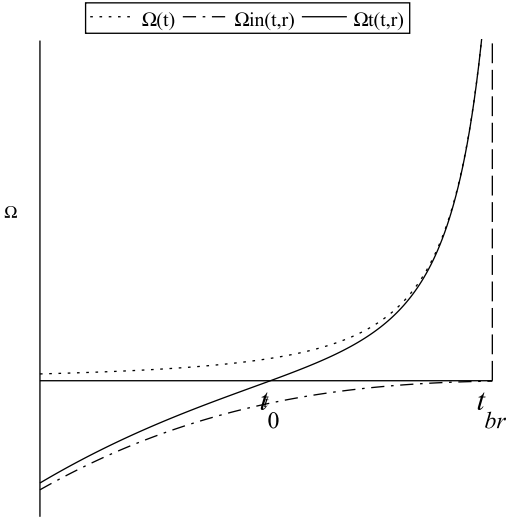

(a) $r_{0}<r_{c}<r_{*}^{-}$

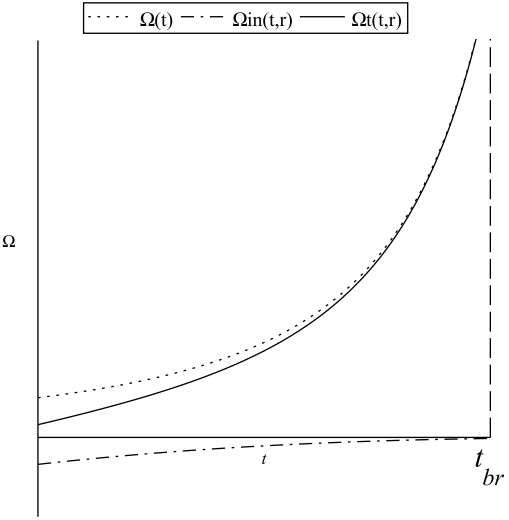

(b) $r_{c}>r_{*}^{-}$

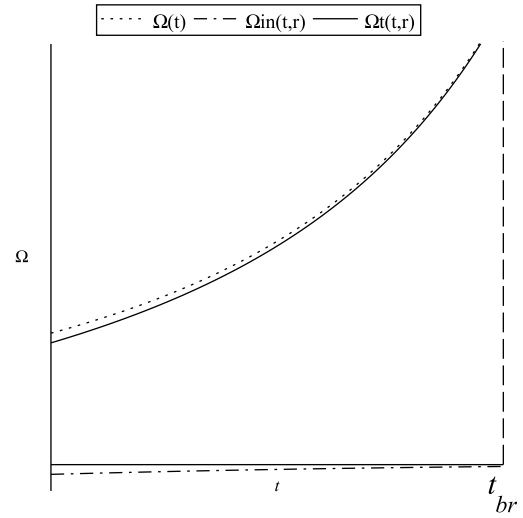

(c) $r_{c} \geq r_{0}^{-}\left(r_{*}^{-}<r_{0}\right)$

FIG. 3: These figures show, for wormhole models with $\omega_{r}>0$ and $\omega<-1$, the qualitative behavior of the dimensionless energy density for the isotropic component $\Omega(t)$ (dotted line), anisotropic matter $\Omega_{i n}\left(t, r_{c}\right)$ (dash-dotted line) and the total matter content $\Omega_{T}\left(t, r_{c}\right)$ (solid line) as a function of the time $t$, at a constant value of the radial coordinate $r_{c}$. The comoving time varies from 0 to the big rip time $t_{b r}$. In this case always the isotropic component dominates over the anisotropic one. The total energy density vanishes at $t=t_{0}$. For Fig. 3(a) we have taken $0<t_{0}<t_{b r}$, while for Figs $3(\mathrm{~b})$ and $3(\mathrm{c}) t_{0}<0$. Here $r_{*}^{-}=r_{0}\left(r_{0}^{2} \omega_{r} a_{0}^{2} \rho_{0}\right)^{-\omega_{r} /\left(1+3 \omega_{r}\right)}$. Note that in this case the anisotropic energy density is always negative.

as a bulk viscosity within a thermodynamical approach. The bulk viscosity introduces dissipation by only redefining the effective pressure, $P_{\text {eff }}$, according to

$$
P_{\text {eff }}=p+\Pi=p-3 \xi H,
$$

where $\Pi=\Pi(t)$ is the bulk viscous pressure, $\xi=\xi(t)$ is the bulk viscosity coefficient and $H$ is the Hubble parameter.

In this case the Friedmann equations (7) and (8), with $k=0$ and $\kappa=8 \pi G=1$, take the form

$$
\begin{array}{r}
3 H^{2}=\rho+\Lambda, \\
\dot{\rho}+3 H(\rho+p+\Pi)=0 .
\end{array}
$$

The violation of DEC is expressed by the relation $\rho+p+$ $\Pi<0$. This condition implies an increasing energy density of the isotropic fluid filling the evolving wormhole, for a positive bulk viscosity coefficient. The condition $\xi>0$ guaranties a positive entropy production and, in consequence, no violation of the second law of the thermodynamics [27]. 
We shall assume that the viscous component obeys the state equation (12), hence from Eq. (23) we have that $P_{e f f}=\omega \rho-3 \xi H$. Thus from Eqs. (24) and (25) we obtain the following evolution equation for $\mathrm{H}$ :

$$
2 \dot{H}+3(\omega+1) H^{2}=3 \xi H+(\omega+1) \Lambda .
$$

From this equation we obtain for $\Lambda=0$ that

$$
H(t)=\frac{e^{\frac{3}{2} \int \xi(t) d t}}{C+\frac{3}{2}(\omega+1) \int e^{\frac{3}{2} \int \xi(t) d t} d t} .
$$

Thus for $\omega \neq-1$ the scale factor is given by

$a(t)=D\left(C+\frac{3}{2}(\omega+1) \int e^{\frac{3}{2} \int \xi(t) d t} d t\right)^{2 /(3(\omega+1))}$,

while for $\omega=-1$ it may be written as

$$
a(t)=D e^{C \int e^{\frac{3}{2} \int \xi(t) d t} d t,}
$$

where $C$ and $D$ are integration constants. In general, for the considered case, the solution may be written through $\xi(t)$ or $a(t)$ because there are three independent equations for the four unknown functions $a(t), \rho(t), \xi(t)$ and $p(t)$. In our case we have written the solution through the bulk viscosity $\xi(t)$. It is worth to mention that for a given $a(t)$ we can write $H$ and then obtain the expressions for the energy density from Eq. (24) and the bulk viscosity from Eq. (26).

It becomes clear that for $\xi=0$ and $\omega \neq-1$ we obtain from Eq. (28) the solution (13) discussed in the previous section, while for a vanishing bulk viscosity the de Sitter scale factor $a(t)=e^{H_{0} t}$ is obtained for $\rho=-p=$ const.

On the other hand, note that from Eq. (24) and Eq. (25) we may write that

$$
\frac{\ddot{a}}{a}=\dot{H}+H^{2}=-\frac{1}{6}\left(\rho+3 P_{e f f}\right)+\frac{\Lambda}{3} .
$$

Thus the condition for an expansion with constant velocity is given by $\rho+3 P_{\text {eff }}=2 \Lambda$. By taking into account Eq. (23) we may write

$$
\xi=\frac{1}{9 H}((1+3 \omega) \rho-2 \Lambda) .
$$

Note that for $\Lambda=0$, and by taking into account Eq. (24), we conclude that in order to have dynamic wormholes expanding with constant velocity the bulk viscosity must be given by

$$
\xi=\frac{(1+3 \omega)}{3 \sqrt{3}} \rho^{1 / 2} .
$$

In this case we see that a necessary condition to have a positive bulk viscosity coefficient is that $\omega>-1 / 3$.

Now we shall consider specific viscous phantom evolving wormhole models.

\section{A. Wormhole models with constant bulk viscosity}

Let us now consider wormhole models with a vanishing cosmological constant and a bulk viscosity given by

$$
\xi(t)=\xi_{0}=\text { const. }
$$

For $\omega \neq-1$, the Eq. (28) allows us to write the scale factor in the form

$$
a(t)=a_{0}\left(1+\frac{H_{0}}{\xi_{0}}(\omega+1)\left(e^{3 \xi_{0} t / 2}-1\right)\right)^{2 /(3(\omega+1))},
$$

from which we obtain that the homogeneous and isotropic energy density is given by

$$
\rho(t)=\frac{3 H_{0}^{2} e^{3 \xi_{0} t}}{\left(1+\frac{H_{0}}{\xi_{0}}(\omega+1)\left(e^{3 \xi_{0} t / 2}-1\right)\right)^{2}} .
$$

Then the anisotropic and inhomogeneous matter component takes the following form:

$$
-\frac{\left(\frac{r}{r_{0}}\right)^{-\left(1+3 \omega_{r}\right) / \omega_{r}} \kappa \rho_{i n}(t, r)=}{r_{0}^{2} \omega_{r} a_{0}^{2}\left(1+\frac{H_{0}}{\xi_{0}}(\omega+1)\left(e^{3 \xi_{0} t / 2}-1\right)\right)^{4 /(3(\omega+1))}} .
$$

As in the previous section, for $\omega<-1$ we have a future singularity at a finite value of the comoving proper time $t_{b r}$ since $a(t) \longrightarrow \infty, \rho(t) \longrightarrow \infty$ and $p \longrightarrow-\infty$ at $t_{b r}=\frac{2}{3 \xi_{0}} \ln \left(1-\frac{\xi_{0}}{H_{0}(\omega+1)}\right)>0$. In this case at $\mathrm{t}=t_{b r}$ the energy density of the anisotropic matter threading the wormhole vanishes since if $\omega_{r}>0$ or $\omega_{r}<-1$ we obtain that $\rho_{i n}\left(t_{b r}, r\right)=0$ for any $r \geq r_{0}$.

If we consider the total energy density $\rho_{T}$, given now by Eqs. (17), (35) and (36), we conclude that for $\omega<-1$ $\rho_{T} \longrightarrow \infty, p_{T r} \longrightarrow \infty$ and $p_{T l} \longrightarrow \infty$ at $t=t_{b r}$. Thus, this future singularity is characterized by diverging scale factor, total energy density and total pressures, but with a well behaved bulk viscosity, since $\xi$ is constant during all evolution.

Notice that for the $\omega=-1$ branch solution we obtain from Eq. (29) that the scale factor is given by

$$
a(t)=D e^{\frac{2 C e^{\frac{3}{2} \xi_{0} t}}{3 \xi_{0}}} .
$$

In this case the model is characterized by an accelerated expansion and does not end in a future singularity.

\section{B. Accelerating wormhole models with $\xi \sim \rho^{1 / 2}$}

Another interesting example in this line is obtained for a bulk viscosity given by $\xi=\alpha \rho^{1 / 2}$, where $\alpha$ is a 
constant parameter. In this case, for any value of the state parameter $\omega$ and $\Lambda=0$, the integration of Eq. (26) allows us to write

$$
H=\frac{H_{0}}{1+\frac{3}{2} H_{0}(\omega+1-\sqrt{3} \alpha) t},
$$

where $H_{0}=H(t=0)$. Thus, the scale factor becomes

$$
a(t)=a_{0}\left(1+\frac{3}{2} H_{0}(\omega+1-\sqrt{3} \alpha) t\right)^{\frac{2}{3(\omega+1-\sqrt{3} \alpha)}}
$$

with $a_{0}=a(t=0)$. The energy density of the isotropic component takes the form

$$
\rho(t)=\frac{3 H_{0}^{2}}{\left(1+\frac{3}{2} H_{0}(\omega+1-\sqrt{3} \alpha) t\right)^{2}},
$$

while the bulk viscosity and the energy density of the anisotropic and inhomogeneous fluid are given by

$$
\begin{gathered}
\xi(t)=\frac{\alpha \sqrt{3} H_{0}}{1+\frac{3}{2} H_{0}(\omega+1-\sqrt{3} \alpha) t}, \\
-\frac{\left(\frac{r}{r_{0}}\right)^{-\left(1+3 \omega_{r}\right) / \omega_{r}} \quad \kappa \rho_{\text {in }}(t, r)=}{r_{0}^{2} \omega_{r} a_{0}^{2}\left(1+\frac{3}{2} H_{0}(\omega+1-\sqrt{3} \alpha) t\right)^{\frac{4}{3(\omega+1-\sqrt{3} \alpha)}}},
\end{gathered}
$$

respectively.

By demanding that

$$
\sqrt{3} \alpha>\omega+1
$$

the scale factor, the isotropic energy density and pressure blow up to infinity at a finite time

$$
t_{b r}=\frac{2 H_{0}^{-1}}{3(\sqrt{3} \alpha-(\omega+1))}>0,
$$

and then we have the occurrence of a future singularity. As in the previous case, at $\mathrm{t}=t_{b r}$ the energy density and pressures of the anisotropic matter threading the wormhole vanishes for any $r \geq r_{0}$.

By considering the total energy density $\rho_{T}$, given now by Eqs. (17), (40) and (42), we conclude that for the constraint (43) we have that $\rho_{T} \longrightarrow \infty, p_{T r} \longrightarrow \infty$ and $p_{T l} \longrightarrow \infty$ at $t=t_{b r}$. Thus, this future singularity is characterized by diverging scale factor, total energy density and total pressures, and diverging too bulk viscosity (41), since it blows up at the time $t_{b r}$. In conclusion, in these viscous expanding wormhole models the scale factor, the total energy density, the total pressures and the bulk viscosity blow up at the finite time $t_{b r}$, so this finite-time future singularity is of a Big Rip type.

It is interesting to note that the constraint (43) implies that if the bulk viscosity is positive, i.e. $\alpha>0$, we can have a future singularity also for $\omega \geq-1$. Thus we have a big rip singularity not only for viscous phantom energy, but also for viscous dark energy, and even for standard viscous matter (see Figs. 4(a) and 4(b). Clearly all these models have an accelerated expansion. It is easy to show that in order to have models expanding with constant velocity we must require that $\frac{2}{3(\omega+1-\sqrt{3} \alpha)}=1$. This implies that $\alpha=(1+3 \omega) / 3 \sqrt{3}$ in agreement with Eq. (32).

\section{CONCLUSIONS AND FURTHER COMMENTS}

This paper deals with inhomogeneous and anisotropic spacetimes, ending in a future singularity at a finite value of the proper time, and filled with an inhomogeneous and anisotropic fluid and another isotropic and homogeneously distributed super-quintessence fluid. The studied solutions describe evolving wormholes for which the rate of expansion is determined by the phantom energy, while the inhomogeneous and anisotropic component threads and sustains the wormhole. The main purpose of this work is to present analytic wormhole models exhibiting a Big Rip during its evolution. Three independent cosmological models are explored.

In the first model it turns out that, the isotropic and homogeneous component is a barotropic phantom fluid. For these evolving wormholes the scale factor, the total energy density and the total pressures blow up at a finite proper time, so this finite-time future singularity is of a Big Rip type. In the second wormhole configuration the isotropic and homogeneous component is a viscous phantom fluid with a constant bulk viscosity. Now, the future singularity is characterized by diverging scale factor, total energy density and total pressures, but with a well behaved bulk viscosity, due to the constant character of $\xi$ during all evolution. In the latter model the isotropic and homogeneous component is a viscous phantom fluid with a bulk viscosity of the form $\xi \sim \rho^{1 / 2}$. For these viscous dynamic wormholes the scale factor, the total energy density, the total pressures and the bulk viscosity blow up at a finite proper time, so this future singularity is also of a Big Rip type. It must be added that this third model allows us to consider Big Rip wormholes not only for a viscous super-quintessence energy. Effectively, if $\xi>0$, we can have a Big Rip also for viscous dark energy (i.e. for $-1<\omega<-1 / 3$ ), and even for standard viscous matter (i.e. for $\omega>-1 / 3$ ). If $\alpha<0$ the Big Rip is avoided. However in this case the bulk viscosity becomes negative and, in consequence, the second law of the thermodynamics is not fulfilled.

Notice that, in all considered here solutions the mixed component of the energy-momentum tensor $T_{t r}$ vanishes. This means that there is no radial energy flow and no accretion onto the wormhole of phantom energy from the cosmic fluid. Thus the mechanism by which the big trip could be achieved is out of the possibilities for these wormhole models [24]. 


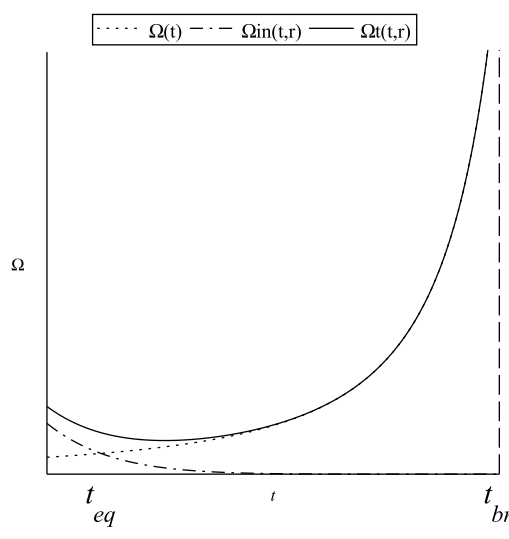

(a) $\omega_{r}<-1$

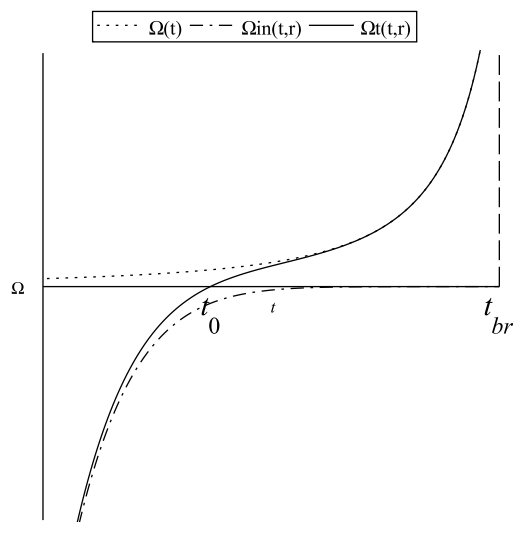

(b) $\omega_{r}>0$

FIG. 4: These figures show, for viscous wormholes with $\xi \sim \rho^{1 / 2}$, the qualitative behavior of the dimensionless energy density for the isotropic component $\Omega(t)$ (dotted line), anisotropic matter $\Omega_{i n}\left(t, r_{c}\right)$ (dash-dotted line) and the total matter content $\Omega_{T}\left(t, r_{c}\right)$ (solid line) as a function of the time $t$, at a constant value of the radial coordinate $r_{c}$. The comoving time varies from 0 to the big rip time $t_{b r}$. For wormholes defined by $\omega_{r}<-1$, we can have a similar behavior for all values of the state parameter $\omega$, i.e. for $\omega<-1,-1<\omega<-1 / 3$ and $\omega>-1 / 3$, as is shown in Fig. 4(a) Here $t_{\text {eq }}$ represents the time at which $\Omega_{i n}\left(t, r_{c}\right)=\Omega(t)$ and the big rip singularity is present even if $\omega>-1$. For wormholes defined by $\omega_{r}>0$ we have again a similar behavior for all values of the state parameter $\omega$, i.e. for $\omega<-1,-1<\omega<-1 / 3$ and $\omega>-1 / 3$, as is shown in Fig. 4(b). Note that in this case the anisotropic energy density is always negative. The big rip singularity is also present even if $\omega>-1$ and $t_{0}$ represents the time at which $\Omega_{T}\left(t, r_{c}\right)=0$.

Lastly, we want to state that all obtained here results on future singularities are applicable also for flat FRW cosmological models, since all the discussed wormhole solutions are asymptotically flat FRW cosmologies.

\section{ACKNOWLEDGEMENTS}

This work was supported by CONICYT through Grant FONDECYT N ${ }^{0} 1080530$ and by the Dirección de Inves- tigación de la Universidad del Bio-Bío through grants $\mathrm{N}^{0}$ DIUBB 121007 2/R and N0 GI121407/VBC (MC).
[1] D. A. Howell, M. Sullivan, P. E. Nugent, R. S. Ellis, A. J. Conley, D. Le Borgne, R. G. Carlberg and J. Guy et al., Nature 443, 308 (2006); A. G. Riess et al. [Supernova Search Team Collaboration], Astrophys. J. 607, 665 (2004); J. L. Tonry et al. [Supernova Search Team Collaboration], Astrophys. J. 594, 1 (2003); S. Perlmutter et al. [Supernova Cosmology Project Collaboration], Astrophys. J. 517, 565 (1999); A. G. Riess et al. [Supernova Search Team Collaboration], Astron. J. 116, 1009 (1998).

[2] E. J. Copeland, M. Sami and S. Tsujikawa, Int. J. Mod. Phys. D 15, 1753 (2006).

[3] A. V. Astashenok, S. 'i. Nojiri, S. D. Odintsov and R. J. Scherrer, Phys. Lett. B 713, 145 (2012); R. A. Sussman and G. Izquierdo, Class. Quant. Grav. 28, 045006 (2011); R. -J. Yang and S. N. Zhang, Mon. Not. Roy. Astron. Soc. 407, 1835 (2010); A. Kurek and M. Szydlowski, Astrophys. J. 675, 1 (2008); V. Sahni, Lect. Notes Phys. 653, 141 (2004); M. S. Turner and M. J. White, Phys. Rev. D 56, 4439 (1997).
[4] G. Olivares, F. Atrio-Barandela and D. Pavon, Phys. Rev. D 71, 063523 (2005); V. F. Cardone, A. Troisi and S. Capozziello, Phys. Rev. D 72, 043501 (2005); T. Matos and L. A. Urena-Lopez, Phys. Rev. D 63, 063506 (2001); E. J. Copeland, N. J. Nunes and F. Rosati, Phys. Rev. D 62, 123503 (2000); P. F. Gonzalez-Diaz, Phys. Rev. D 62, 023513 (2000).

[5] M. U. Farooq, M. Jamil and M. A. Rashid, Int. J. Theor. Phys. 49, 2278 (2010); N. Cruz, P. F. Gonzalez-Diaz, A. Rozas-Fernandez and G. Sanchez, Phys. Lett. B 679, 293 (2009); L. P. Chimento, Phys. Rev. D 69, 123517 (2004); T. Chiba, Phys. Rev. D 66, 063514 (2002).

[6] K. Karami, S. Ghaffari and J. Fehri, Eur. Phys. J. C 64, 85 (2009); H. Mohseni Sadjadi and M. Alimohammadi, Phys. Rev. D 74, 043506 (2006); J. Kujat, R. J. Scherrer and A. A. Sen, Phys. Rev. D 74, 083501 (2006); L. Perivolaropoulos, Phys. Rev. D 71, 063503 (2005).

[7] O. -C. Stoica, Commun. Theor. Phys. 58, 613 (2012); G. Niz and N. Turok, Phys. Rev. D 75, 026001 (2007); C. Cattoen and M. Visser, Class. Quant. Grav. 22, 4913 
(2005); J. M. M. Senovilla, Phys. Rev. Lett. 64, 2219 (1990); G. L. Murphy, Phys. Rev. D 8, 4231 (1973).

[8] P. Singh, Class. Quant. Grav. 26, 125005 (2009); M. P. Dabrowski and A. Balcerzak, gr-qc/0701056

[9] S. Nojiri, S.D. Odintsov and S. Tsujikawa, Phys. Rev. D 71, 063004 (2005).

[10] M.S. Morris and K.S. Thorne, Am. J. Phys. 56, 395 (1988); M.S. Morris, K.S. Thorne and U. Yurtsever, Phys. Rev. Lett. 61, 1446 (1988); M. Visser, Lorentzian Wormholes: From Einstein to Hawking, (AIP, New York, 1995).

[11] A. Anabalon and A. Cisterna, Phys. Rev. D 85, 084035 (2012); G. A. S. Dias and J. P. S. Lemos, Phys. Rev. D 82, 084023 (2010); A. B. Balakin, J. P. S. Lemos and A. E. Zayats, Phys. Rev. D 81, 084015 (2010); M. Jamil, P. K. F. Kuhfittig, F. Rahaman and S. A. Rakib, Eur. Phys. J. C 67, 513 (2010); M. Jamil and M. U. Farooq, Int. J. Theor. Phys. 49, 835 (2010); E. F. Eiroa, Phys. Rev. D 80, 044033 (2009); M. Cataldo, P. Salgado and P. Minning, Phys. Rev. D 66, 124008 (2002).

[12] M. Cataldo, P. Meza and P. Minning, Phys. Rev. D 83, 044050 (2011); S. H. Hendi, J. Math. Phys. 52, 042502 (2011); S. H. Hendi, Can. J. Phys. 89, 281 (2011); I. Bochicchio and V. Faraoni, Phys. Rev. D 82, 044040 (2010); M. Jamil and M. Akbar, arXiv:0911.2556 [hep-th]; M. Jamil, arXiv:0909.3689 [gr-qc]; J. Hansen, D. i. Hwang and D. h. Yeom, JHEP 0911, 016 (2009); M. H. Dehghani and S. H. Hendi, Gen. Rel. Grav. 41, 1853 (2009); A. DeBenedictis, R. Garattini and F. S. N. Lobo, Phys. Rev. D 78, 104003 (2008); P. F. Gonzalez-Diaz, Phys. Rev. D 68, 084016 (2003).

[13] F. S. N. Lobo and M. Visser, Class. Quant. Grav. 21, 5871 (2004).

[14] S. Kar, Phys. Rev D 49, 862 (1994); S. Kar and D. Sahdev, Phys. Rev D 53, 722 (1996).

[15] H. Maeda, T. Harada and B.J. Carr, Phys. Rev. D 79, 044034 (2009).

[16] M. Cataldo and S. del Campo, Phys. Rev. D 85, 104010 (2012).

[17] M. Cataldo, P. Mella, P. Minning and J. Saavedra, Phys. Lett. B 662, 314 (2008); A. Banijamali, B. Fazlpour, Phys. Lett. B703, 366-369 (2011); S. Chattopadhyay, U. Debnath, Astrophys. Space Sci. 326, 155-158 (2010); H. Farajollahi, N. Mohamadi, H. Amiri, Mod. Phys. Lett. A25, 2579-2589 (2010); Y. -F. Cai, J. Wang, Class.
Quant. Grav. 25, 165014 (2008); I. H. Brevik, Gen. Rel Grav. 38, 1317-1328 (2006).

[18] M. Jamil, P. K. F. Kuhfittig, F. Rahaman and S. .ARakib, Eur. Phys. J. C 67, 513 (2010); M. Jamil and M. U. Farooq, Int. J. Theor. Phys. 49, 835 (2010); J. A. Gonzalez, F. S. Guzman, N. Montelongo-Garcia and T. Zannias, Phys. Rev. D 79, 064027 (2009); M. Jamil, U. Farooq and M. A. Rashid, Eur. Phys. J. C 59, 907 (2009); K. A. Bronnikov and A. A. Starobinsky, JETP Lett. 85, 1 (2007); F. Rahaman, M. Kalam, B. C. Bhui and S. Chakraborty, Phys. Scripta 76, 56 (2007); P. K. F. Kuhfittig, Class. Quant. Grav. 23, 5853 (2006); O. B. Zaslavskii, Phys. Rev. D 72, 061303 (2005).

[19] F. S. N. Lobo, Phys. Rev. D 71, 084011 (2005); S. V. Sushkov, Phys. Rev. D 71, 043520 (2005).

[20] M. Cataldo, P. Labraña, S. del Campo, J. Crisostomo and P. Salgado, Phys. Rev. D 78, 104006 (2008); M. Cataldo, S. del Campo, P. Minning and P. Salgado, Phys. Rev. D 79, 024005 (2009).

[21] A. R. El-Nabulsi, Gen. Rel. Grav. 43, 261 (2011).

[22] V. Faraoni and W. Israel, Phys. Rev. D 71, 064017 (2005)

[23] A. V. Astashenok, S. Nojiri, S. D. Odintsov and A. V. Yurov, Phys. Lett. B 709, 396 (2012); A. V. Yurov, A. V. Astashenok and V. A. Yurov, Astrophys. Space Sci. 342, 1 (2012); P. F. Gonzalez-Diaz, Phys. Lett. B 635, 1 (2006); J. A. Jimenez Madrid, Phys. Lett. B 634, 106 (2006); A. V. Yurov, P. Martin Moruno and P. F. Gonzalez-Diaz, Nucl. Phys. B 759, 320 (2006); P. F. Gonzalez-Diaz, Phys. Lett. B 632, 159 (2006); P. F. Gonzalez-Diaz and J. A. Jimenez-Madrid, Phys. Lett. B 596, 16 (2004); P. F. Gonzalez-Diaz, Phys. Rev. Lett. 93, 071301 (2004)

[24] V. Faraoni, Phys. Lett. B 647, 309 (2007).

[25] R. Colistete, J. C. Fabris, J. Tossa and W. Zimdahl, Phys. Rev. D 76, 103516 (2007); A. Pradhan and P. Pandey, Astrophys. Space Sci. 301, 127 (2006); I. H. Brevik, O. Gorbunova and Y. A. Shaido, Int. J. Mod. Phys. D 14, 1899 (2005); I. H. Brevik and O. Gorbunova, Gen. Rel. Grav. 37, 2039 (2005); W. Zimdahl, Phys. Rev. D 53, 5483 (1996).

[26] M. Cataldo, N. Cruz and S. Lepe, Phys. Lett. B 619, 5 (2005).

[27] W. Zimdahl and D. Pavón, Phys. Rev. 61, 108301 (2000). 